\title{
Green-Schwarz Mechanism for String Dualities
}

\author{
Camille Eloy $\odot,{ }^{1}$ Olaf Hohm, ${ }^{2}$ and Henning Samtleben $\oplus^{1}$ \\ ${ }^{1}$ Univ Lyon, Ens de Lyon, Univ Claude Bernard, CNRS, Laboratoire de Physique, F-69342 Lyon, France \\ ${ }^{2}$ Institute for Physics, Humboldt University Berlin, Zum Großen Windkanal 6, D-12489 Berlin, Germany
}

(Received 13 December 2019; accepted 21 January 2020; published 6 March 2020)

\begin{abstract}
We determine the complete spacetime action to first order in $\alpha^{\prime}$ for the massless fields of bosonic string theory compactified on a $d$-dimensional torus. A fully systematic procedure is developed that brings the action into a minimal form in which all fields apart from the metric enter only with first-order derivatives. $T$ duality implies that this action must have a global $\mathrm{O}(d, d, \mathbb{R})$ symmetry, and we show that this requires a Green-Schwarz type mechanism for $\alpha^{\prime}$-deformed $\mathrm{O}(d, d, \mathbb{R})$ transformations. In terms of a frame formalism with $\mathrm{GL}(d) \times \mathrm{GL}(d)$ gauge symmetry this amounts to a modification of the three-form curvature by a Chern-Simons term for composite gauge fields.
\end{abstract}

DOI: 10.1103/PhysRevLett.124.091601

String theory is a particularly promising candidate for a consistent theory of quantum gravity, but it is fair to say that its underlying principles remain elusive. An important clue is that, even classically, string theory modifies general relativity in two significant ways. First, string theory features novel dualities which imply that theories defined on seemingly different backgrounds are actually equivalent. Second, the classical field equations receive an infinite number of higher-derivative corrections governed by the dimensionful (inverse) string tension $\alpha^{\prime}$. Whatever the fundamental formulation of string or M theory may be, it seems clear that it would have to accommodate these two features as core principles. In this Letter we report on results for a fully systematic procedure to determine the duality invariant spacetime action for massless string fields to higher order in $\alpha^{\prime}$ and point out a curious interplay between string dualities and $\alpha^{\prime}$ corrections. We discuss only the main results; the technical details will be presented in Ref. [1].

The simplest duality shared by all closed string theories is $T$ duality. It states that string backgrounds containing a $d$-dimensional torus are mapped under the group $\mathrm{O}(d, d, \mathbb{Z})$ to physically equivalent backgrounds. This duality includes, for a single circle, the inversion of the radius $R \rightarrow \alpha^{\prime} / R$. The $T$-duality property of closed string theory implies that the spacetime action for the massless string fields on such backgrounds features a global $\mathrm{O}(d, d, \mathbb{R})$ symmetry [2]. To lowest order in $\alpha^{\prime}$ this symmetry was first displayed in the cosmological setting (reduction to one

Published by the American Physical Society under the terms of the Creative Commons Attribution 4.0 International license. Further distribution of this work must maintain attribution to the author(s) and the published article's title, journal citation, and DOI. Funded by SCOAP ${ }^{3}$. dimension) in Ref. [3] and later generalized to arbitrary $d$ in Ref. [4]. Subsequent seminal work by Meissner revealed an $\mathrm{O}(d, d, \mathbb{R})$ invariance to first order in $\alpha^{\prime}$ in the cosmological setting, with the group action being undeformed thanks to a judicious choice of field variables [5]. In Ref. [6] the case of a single circle was investigated, while Ref. [7] includes a general torus but truncates to "internal" field degrees of freedom.

More recently, $\alpha^{\prime}$ corrections have been investigated in the extended framework of double field theory [8-10], which is the duality-covariant formulation of spacetime actions before compactification. Notably, two unexpected new features appear: (i) the gauge transformations need to be $\alpha^{\prime}$ corrected, and (ii) there is no background-independent formulation in terms of the familiar $\mathrm{O}(d, d, \mathbb{R})$ matrix encoding metric and $B$ field [11-16]. Rather, the general formulation of double field theory to first order in $\alpha^{\prime}$ employs a frame formalism with $\alpha^{\prime}$ corrected tangent space transformations. While in the dimensionally reduced theories determined so far there is a choice of field basis for which the $\mathrm{O}(d, d, \mathbb{R})$ action is undeformed at order $\alpha^{\prime}$, it has remained an open question whether $\alpha^{\prime}$ deformations of $\mathrm{O}(d, d, \mathbb{R})$ may be required in general dimensionally reduced theories. The reduction of the $\alpha^{\prime}$-deformed double field theory has been investigated in Ref. [17], however, without extracting the consequences for the realization of $\mathrm{O}(d, d, \mathbb{R})$ in the dimensional reduction of conventional (nonextended) theories.

In this Letter we give the complete action to first order in $\alpha^{\prime}$ for arbitrary $d$ and show that in general the $\mathrm{O}(d, d, \mathbb{R})$ transformations are $\alpha^{\prime}$ deformed according to a novel Green-Schwarz type mechanism. The original GreenSchwarz mechanism, which triggered the first superstring revolution, is needed to show that gravitational and gauge anomalies can be canceled, for gauge groups $\mathrm{SO}(32)$ or $E_{8} \times E_{8}$, by an $\alpha^{\prime}$ deformation of the gauge transformations 
of the (singlet) $B$ field, thereby modifying the classical (tree-level) theory [18]. Similarly, we show here that $\mathrm{O}(d, d, \mathbb{R})$ invariance of the spacetime action reduced on a $d$-dimensional torus requires a nontrivial transformation for the singlet $B$ field already classically, with a ChernSimons type modification of the 3-form curvature. This suggests that Green-Schwarz type mechanisms may be much more ubiquitous than expected.

We consider the two-derivative effective action for the bosonic string in $D+d$ dimensions, with metric $\hat{g}_{\hat{\mu} \hat{\nu}}$, antisymmetric Kalb-Ramond field $\hat{B}_{\hat{\mu} \hat{\nu}}$ and the dilaton $\hat{\phi}$

$$
\hat{I}_{0}=\int d^{D+d} X \sqrt{-\hat{g}} e^{-\hat{\phi}}\left(\hat{R}+\partial_{\hat{\mu}} \hat{\phi} \partial^{\hat{\mu}} \hat{\phi}-\frac{1}{12} \hat{H}^{2}\right),
$$

where indices $\hat{\mu}$ run over the $(D+d)$ dimensional space, and $\hat{H}^{2}=\hat{H}^{\hat{\mu} \hat{\nu} \hat{\rho}} \hat{H}_{\hat{\mu} \hat{\nu} \hat{\rho}}$ with the field strength $\hat{H}_{\hat{\mu} \hat{\nu} \hat{\rho}}=$ $3 \partial_{[\hat{\mu}} \hat{B}_{\hat{\nu} \hat{\rho}]}$. For compactification on the $d$-dimensional torus, we use the index split

$$
X^{\hat{\mu}}=\left(x^{\mu}, y^{m}\right), \quad \mu \in \llbracket 1, D \rrbracket, \quad m \in \llbracket 1, d \rrbracket,
$$

and drop the field dependance on the internal coordinates $y^{m}$. For the metric $\hat{g}_{\hat{\mu} \hat{\nu}}$, we consider the standard KaluzaKlein ansatz

$$
\hat{g}_{\hat{\mu} \hat{\nu}}=\left(\begin{array}{cc}
g_{\mu \nu}+A_{\mu}^{(1) p} G_{p q} A_{\nu}^{(1) q} & A_{\mu}^{(1) p} G_{p n} \\
G_{m p} A_{\nu}^{(1) p} & G_{m n}
\end{array}\right),
$$

in terms of the $D$-dimensional metric $g_{\mu \nu}$, Kaluza-Klein vector fields $A_{\mu}^{(1) m}$ and the internal metric $G_{m n}$. Similarly, for the 2 -form $\hat{B}_{\hat{\mu} \hat{\nu}}$, we use the ansatz

$$
\begin{aligned}
\hat{B}_{m n} & =B_{m n}, \\
\hat{B}_{\mu m} & =A_{\mu m}^{(2)}-A_{\mu}^{(1) n} B_{m n}, \\
\hat{B}_{\mu \nu} & =B_{\mu \nu}-A_{[\mu}^{(1) m} A_{\nu] m}^{(2)}+A_{\mu}^{(1) m} B_{m n} A_{\nu}^{(1) n},
\end{aligned}
$$

in terms of $D$-dimensional scalars $B_{m n}$, vector fields $A_{\mu m}^{(2)}$, and a 2-form $B_{\mu \nu}$. After dimensional reduction, the action (1) may be cast into the manifestly $\mathrm{O}(d, d, \mathbb{R})$ invariant form [4]

$$
\begin{aligned}
I_{0}= & \int d^{D} x \sqrt{-g} e^{-\Phi}\left(R+\partial_{\mu} \Phi \partial^{\mu} \Phi-\frac{1}{12} H^{2}\right. \\
& \left.+\frac{1}{8} \operatorname{Tr}\left(\nabla_{\mu} \mathcal{H} \nabla^{\mu} \mathcal{H}^{-1}\right)-\frac{1}{4} \mathcal{F}_{\mu \nu}{ }^{M} \mathcal{H}_{M N} \mathcal{F}^{\mu \nu N}\right) .
\end{aligned}
$$

Here, $\Phi=\hat{\phi}-\frac{1}{2} \log \left[\operatorname{det}\left(G_{m n}\right)\right]$ is the shifted dilaton and the $\mathrm{O}(d, d, \mathbb{R})$ matrix $\mathcal{H}$ is given by

$$
\mathcal{H}_{M N}=\left(\begin{array}{cc}
G_{m n}-B_{m p} G^{p q} B_{q n} & B_{m p} G^{p n} \\
-G^{m p} B_{p n} & G^{m n}
\end{array}\right) .
$$

The vectors $A_{\mu}^{(1) m}$ and $A_{\mu m}^{(2)}$ are combined into an $\mathrm{O}(d, d, \mathbb{R})$ vector

$$
\mathcal{A}_{\mu}{ }^{M}=\left(\begin{array}{c}
A_{\mu}^{(1) m} \\
A_{\mu m}^{(2)}
\end{array}\right),
$$

and $\mathcal{F}_{\mu \nu}{ }^{M}=2 \partial_{[\mu} \mathcal{A}_{\nu]}{ }^{M}$ is the associated Abelian field strength. Finally, the $D$-dimensional 3-form $H_{\mu \nu \rho}$ in Eq. (5) is given by

$$
H_{\mu \nu \rho}=3\left(\partial_{[\mu} B_{\nu \rho]}-\frac{1}{2} \mathcal{A}_{[\mu}{ }^{M} \mathcal{F}_{\nu \rho] M}\right) .
$$

Throughout, the $\mathrm{O}(d, d, \mathbb{R})$ indices are raised and lowered using the constant $\mathrm{O}(d, d, \mathbb{R})$-invariant matrix

$$
\eta^{M N}=\left(\begin{array}{cc}
0 & \delta^{m}{ }_{n} \\
\delta_{m}{ }^{n} & 0
\end{array}\right) .
$$

In terms of the covariant objects (6), (7), the action (5) makes the invariance under $\mathrm{O}(d, d, \mathbb{R})$ transformations manifest. The goal of this Letter is the extension of this construction in presence of higher order corrections at first order in $\alpha^{\prime}$.

At first order in $\alpha^{\prime}$, the correction to the action (1) is given by [19]

$$
\begin{aligned}
& \hat{I}_{1}=\frac{\alpha^{\prime}}{4} \int d^{D+d} X \sqrt{-\hat{g}} e^{-\hat{\phi}}\left(\hat{R}_{\hat{\mu} \hat{\nu} \hat{\rho} \hat{\sigma}} \hat{R}^{\hat{\mu} \hat{\nu} \hat{\rho} \hat{\sigma}}-\frac{1}{8} \hat{H}_{\hat{\mu} \hat{\nu}}^{2} \hat{H}^{2 \hat{\mu} \hat{\nu}}\right.
\end{aligned}
$$

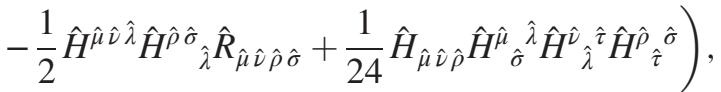

up to field redefinitions. Here, $\hat{R}_{\hat{\mu} \hat{\nu} \hat{\rho} \hat{\sigma}}$ is the Riemann tensor, and we defined $\hat{H}_{\hat{\mu} \hat{\nu}}^{2} \equiv \hat{H}^{\hat{\mu} \hat{\rho} \hat{\sigma}} \hat{H}_{\hat{\rho} \hat{\rho}}$. In the following, we compactify this action on the $d$-dimensional torus and seek to bring it into a manifestly $\mathrm{O}(d, d, \mathbb{R})$ invariant form. In the presence of higher order corrections this construction appears far less straightforward than in the two-derivative case due to the many potential ambiguities from field redefinitions and partial integrations.

Let us outline our systematics, which generalize those in Refs. $[20,21]$ to arbitrary dimensions. We first determine a basis of independent $\mathrm{O}(d, d, \mathbb{R})$ invariant four-derivative terms. In $D$ dimensions, every four-derivative term carrying the leading two-derivative contribution from the field equations descending from the two-derivative action (5) can be eliminated by a field redefinition in order $\alpha^{\prime}$. Moreover, different four-derivative terms in the Lagrangian can be related by integration by parts. Upon a systematic count, dividing out this freedom, we find that 
at order $\alpha^{\prime}$, there are 61 independent four-derivative terms that can be built from the $\mathrm{O}(d, d, \mathbb{R})$ covariant objects $\mathcal{H}_{M N}, \mathcal{F}_{\mu \nu}{ }^{M}, H_{\mu \nu \rho}, R_{\mu \nu \rho \sigma}$ and their derivatives. By explicit construction, we then show the existence of a distinguished basis, which, except for the $D$-dimensional Riemann tensor, only carries first order derivative terms, i.e., which is polynomial in $\nabla_{\mu} \Phi, \nabla_{\mu} \mathcal{H}_{M N}, \mathcal{F}_{\mu \nu}{ }^{M}, H_{\mu \nu \rho}$, and $R_{\mu \nu \rho \sigma}$.

Next, we compactify separately every term from Eq. (10) with the ansatz (3), (4) and find that all terms carrying second order derivatives can indeed be eliminated by field redefinitions and integrations by part. Generically, these field redefinitions will not be $\mathrm{O}(d, d, \mathbb{R})$ covariant.
The remaining terms then can be matched against the previously determined 61-dimensional $\mathrm{O}(d, d, \mathbb{R})$ invariant basis after breaking the terms of the latter under GL $(d)$ according to Eqs. (6) and (7). Details of this rather lengthy computation will appear in Ref. [1]. As a result, we find that after compactification the action (10) can be brought into the form

$$
\hat{I}_{1} \rightarrow I_{1}+O_{1},
$$

where $I_{1}$ takes the manifestly $\mathrm{O}(d, d, \mathbb{R})$ invariant form

$$
\begin{aligned}
I_{1}= & \frac{1}{4} \alpha^{\prime} \int d^{D} x \sqrt{-g} e^{-\Phi}\left[R_{\mu \nu \rho \sigma} R^{\mu \nu \rho \sigma}-\frac{1}{2} R_{\mu \nu \rho \sigma} H^{\mu \nu \lambda} H_{\lambda}^{\rho \sigma}-\frac{1}{8} H_{\mu \nu}^{2} H^{2 \mu \nu}+\frac{1}{24} H_{\mu \nu \rho} H^{\mu}{ }_{\sigma}{ }^{\lambda} H^{\nu}{ }_{\lambda}{ }^{\tau} H^{\rho}{ }_{\tau}{ }\right. \\
& +\frac{1}{16} \operatorname{Tr}\left(\nabla_{\mu} \mathcal{S} \nabla_{\nu} \mathcal{S} \nabla^{\mu} \mathcal{S} \nabla^{\nu} \mathcal{S}\right)-\frac{1}{32} \operatorname{Tr}\left(\nabla_{\mu} \mathcal{S} \nabla_{\nu} \mathcal{S}\right) \operatorname{Tr}\left(\nabla^{\mu} \mathcal{S} \nabla^{\nu} \mathcal{S}\right)+\frac{1}{8} \mathcal{F}_{\mu \nu}{ }^{M} \mathcal{S}_{M}{ }^{N} \mathcal{F}_{\rho \sigma N} \mathcal{F}^{\mu \rho P} \mathcal{S}_{P}{ } \mathcal{F}^{\nu \sigma}{ }_{Q} \\
& -\frac{1}{2} \mathcal{F}_{\mu \nu}{ }^{M} \mathcal{S}_{M}{ }^{N} \mathcal{F}^{\mu \rho}{ }_{N} \mathcal{F}^{\nu \sigma P} \mathcal{S}_{P} Q \mathcal{F}_{\rho \sigma Q}+\frac{1}{8} \mathcal{F}_{\mu \nu}{ }^{M} \mathcal{F}_{\rho \sigma M} \mathcal{F}^{\mu \rho N} \mathcal{F}^{\nu \sigma}{ }_{N} \\
& -\frac{1}{2} R_{\mu \nu \rho \sigma} \mathcal{F}^{\mu \nu M} \mathcal{S}_{M}{ }^{N} \mathcal{F}^{\rho \sigma}{ }_{N}+\frac{1}{8} H_{\mu \nu}^{2} \operatorname{Tr}\left(\nabla^{\mu} \mathcal{S} \nabla^{\nu} \mathcal{S}\right)-\frac{1}{2} H_{\mu \nu}^{2} \mathcal{F}^{\mu}{ }_{\rho}{ }^{M} \mathcal{S}_{M}{ }^{N} \mathcal{F}^{\nu \rho}{ }_{N}+\frac{1}{4} H^{\mu \nu \lambda} H^{\rho \sigma}{ }_{\lambda} \mathcal{F}_{\mu \rho}{ }^{M} \mathcal{S}_{M}{ }^{N} \mathcal{F}_{\nu \sigma N} \\
& \left.-\frac{1}{2} \mathcal{F}_{\mu \nu}{ }^{M}\left(\mathcal{S} \nabla_{\rho} \mathcal{S} \nabla^{\nu} \mathcal{S}\right)_{M}{ }^{N} \mathcal{F}^{\mu \rho}{ }_{N}+\frac{1}{4} \mathcal{F}^{\mu \rho M} \mathcal{S}_{M}{ }^{N} \mathcal{F}^{\nu}{ }_{\rho N} \operatorname{Tr}\left(\nabla_{\mu} \mathcal{S} \nabla_{\nu} \mathcal{S}\right)-\frac{1}{2} H^{\mu \nu \rho} \mathcal{F}_{\mu \sigma}{ }^{M}\left(\mathcal{S} \nabla_{\nu} \mathcal{S}\right)_{M}{ }^{N} \mathcal{F}_{\rho}{ }^{\sigma}{ }_{N}\right],
\end{aligned}
$$

with $H_{\mu \nu}^{2}=H^{\mu \rho \sigma} H_{\rho \sigma}^{\nu}$, and the matrix $\mathcal{S}$ defined as $\mathcal{S}_{M}{ }^{N}=\mathcal{H}_{M P} \eta^{P N}$. In contrast, the term $O_{1}$ is not $\mathrm{O}(d, d, \mathbb{R})$ invariant, but takes the particular form

$$
O_{1}=\frac{1}{6} \alpha^{\prime} \int d^{D} x \sqrt{-g} e^{-\Phi} H_{\mu \nu \rho} \Omega^{\mu \nu \rho},
$$

with $\Omega_{\mu \nu \rho}$ given by

$$
\begin{aligned}
\Omega_{\mu \nu \rho}= & -\frac{3}{4} \operatorname{Tr}\left(\partial_{[\mu} G^{-1} G \partial_{\nu} G^{-1} \partial_{\rho]} B\right) \\
& +\frac{1}{4} \operatorname{Tr}\left(\partial_{[\mu} B G^{-1} \partial_{\nu} B G^{-1} \partial_{\rho]} B G^{-1}\right) .
\end{aligned}
$$

This 3-form has a remarkable structure owing its existence to the nonvanishing cohomology $H^{4}(M)$ of $M=\mathrm{O}(d, d, \mathbb{R}) /[\mathrm{O}(d) \times \mathrm{O}(d)]$. Although $\Omega_{\mu \nu \rho}$ it is not $\mathrm{O}(d, d, \mathbb{R})$ invariant, its exterior derivative is, as it takes the manifestly invariant form

$$
4 \partial_{[\mu} \Omega_{\nu \rho \sigma]}=\frac{3}{8} \operatorname{Tr}\left(\mathcal{S} \partial_{[\mu} \mathcal{S} \partial_{\nu} \mathcal{S} \partial_{\rho} \mathcal{S} \partial_{\sigma]} \mathcal{S}\right) .
$$

We may thus conclude that the $\mathrm{O}(d, d, \mathbb{R})$ variation of $\Omega_{\mu \nu \rho}$ is a closed 3-form, which locally may be expressed as

$$
\delta \Omega_{\mu \nu \rho}=3 \partial_{[\mu} X_{\nu \rho]},
$$

in terms of a 2-form $X_{\mu \nu}$. This observation together with the particular form of Eq. (13) suggests a Green-Schwarz type mechanism in order to restore $\mathrm{O}(d, d, \mathbb{R})$ invariance of the $D$-dimensional action.

Namely, we may rewrite the result of the compactification of Eqs. (1) and (10) as

$$
\hat{I}_{0}+\hat{I}_{1} \rightarrow \tilde{I}_{0}+\tilde{I}_{1}+\mathcal{O}\left(\alpha^{2}\right),
$$

where $\tilde{I}_{0}, \tilde{I}_{1}$ denote the actions (5) and (12), respectively, in which we have replaced all 3-form curvatures $H_{\mu \nu \rho}$ by the deformation

$$
\widetilde{H}_{\mu \nu \rho} \equiv H_{\mu \nu \rho}-\alpha^{\prime} \Omega_{\mu \nu \rho} .
$$

Indeed, this deformation of the lowest order action (5) precisely produces the noninvariant term (13) at order $\alpha^{\prime}$ while the deformation of Eq. (12) only contributes to higher orders in $\alpha^{\prime}$. As a last step, we observe that the deformed field strength (18) is $\mathrm{O}(d, d, \mathbb{R})$ invariant if the 2-form $B_{\mu \nu}$ acquires a nontrivial variation

$$
\delta B_{\mu \nu}=\alpha^{\prime} X_{\mu \nu},
$$

under $\mathrm{O}(d, d, \mathbb{R})$ transformations, with the right-hand side defined by integrating Eq. (16). With this new anomalous transformation, the resulting theory is then fully $\mathrm{O}(d, d, \mathbb{R})$ invariant to first order in $\alpha^{\prime}$. As expected, this is only true 
for the precise choice of relative coefficients in the $(D+d)$ dimensional action (10). In fact, the same method allows us to prove a stronger result: starting from the most general action of eight independent four-derivative terms in $(D+d)$ dimensions [19], $\mathrm{O}(d, d, \mathbb{R})$ invariance of the reduced action uniquely fixes all the coefficients up to an overall factor.

An explicit calculation of the variation (16) for a general $\mathfrak{o}(d, d, \mathbb{R})$ generator

$$
\alpha_{M}{ }^{N}=\left(\begin{array}{cc}
\mathfrak{a}_{m}{ }^{n} & \mathfrak{b}_{m n} \\
\mathfrak{c}^{m n} & -\mathfrak{a}_{n}{ }^{m}
\end{array}\right),
$$

with $\mathfrak{c}^{m n}$ and $\mathfrak{b}_{m n}$ antisymmetric, yields the explicit form of the new $\mathfrak{v}(d, d, \mathbb{R})$ transformation of $B_{\mu \nu}$ as

$$
X_{\mu \nu}=\frac{1}{2} \operatorname{Tr}\left(\mathfrak{c} \partial_{[\mu}(G+B) G^{-1} \partial_{\nu]}(G+B)\right) .
$$

The 2-form thus acquires new transformations only along the nilpotent $\mathfrak{v}(d, d, \mathbb{R})$ generators $\mathfrak{c}^{m n}$. This is consistent with the fact that all the other $\mathfrak{v}(d, d, \mathbb{R})$ generators have a geometric origin and hence should be a manifest invariance of the dimensionally reduced action. Moreover, with the expression (21), the algebra of $\mathfrak{v}(d, d, \mathbb{R})$ transformations closes on $B_{\mu \nu}$. Crucially, the deformed $\mathfrak{o}(d, d, \mathbb{R})$ action (19) cannot be absorbed into a redefinition of the fields.

The action (12) is in agreement with previous results in the literature, up to field redefinitions. For $D=1$, it reproduces the result of Refs. [5,20]. Once truncated to scalars only (i.e., setting $\mathcal{A}_{\mu}{ }^{M}=0=B_{\mu \nu}, g_{\mu \nu}=\eta_{\mu \nu}$ ), the action (12) reduces to two terms which provide an equivalent compact rewriting of the action given in Ref. [7]. However, the need for the Green-Schwarz type mechanism (19) is not visible in any of these limits, as the mechanism mixes the Kalb-Ramond field $B_{\mu \nu}$ and the scalars $B_{m n}$.

It is instructive to inspect the above Green-Schwarz deformation in view of the $\mathbb{Z}_{2}$ invariance of bosonic string theory that sends $\hat{B} \rightarrow-\hat{B}$. On the $\mathrm{O}(d, d, \mathbb{R})$ matrix (6) this symmetry acts via the matrix $Z$ [10]:

$$
\mathcal{H} \rightarrow Z^{T} \mathcal{H} Z, \quad Z \equiv\left(\begin{array}{cc}
1 & 0 \\
0 & -1
\end{array}\right) .
$$

This matrix satisfies $Z=Z^{-1}=Z^{T}$ but is not $\mathrm{O}(d, d, \mathbb{R})$ valued since the metric (9) transforms as

$$
\eta \rightarrow Z \eta Z^{T}=-\eta
$$

Consequently, the matrix $\mathcal{S}$ obtained from $\mathcal{H}$ by raising of one index transforms as

$$
\mathcal{S} \rightarrow-Z \mathcal{S Z}
$$

Thus, the $\mathrm{O}(d, d, \mathbb{R})$ invariant defined by the right-hand side of Eq. (15) is $\mathbb{Z}_{2}$ odd. This is as it should be for $\mathbb{Z}_{2}$ invariance of the action since $B_{\mu \nu}$ and its field strength are also $\mathbb{Z}_{2}$ odd.

We have seen that the rigid $O(d, d, \mathbb{R})$ transformations need to be $\alpha^{\prime}$ deformed via a Green-Schwarz type mechanism. We now show that the theory can be reformulated, by means of a frame formalism, so that instead the gauge group of local frame transformations gets $\alpha^{\prime}$ deformed. This formulation then follows the standard Green-Schwarz mechanism more closely, albeit with composite gauge fields.

We introduce a frame field $E \equiv\left(E_{M}{ }^{A}\right)$ with inverse $E^{-1} \equiv\left(E_{A}{ }^{M}\right)$ in terms of which the matrix (6) is given by

$$
\mathcal{H}_{M N}=E_{M}{ }^{A} E_{N}{ }^{B} \kappa_{A B}
$$

where flat indices are split as $A=(a, \bar{a})$, and we assume $\kappa_{A B}$ to be block-diagonal with components $\kappa_{a b}$ and $\kappa_{\bar{a} \bar{b}}$. Since we make no further $a$ priori assumption on $\kappa$ there is a local $\mathrm{GL}(d) \times \mathrm{GL}(d)$ frame invariance, acting as

$$
\delta_{\Lambda} E_{A}{ }^{M}=\Lambda_{A}{ }^{B} E_{B}{ }^{M}, \quad \Lambda_{A}{ }^{B}=\left(\begin{array}{cc}
\Lambda_{a}{ }^{b} & 0 \\
0 & \bar{\Lambda}_{\bar{a}}^{\bar{b}}
\end{array}\right) .
$$

Gauge fixing $\kappa_{A B}=\delta_{A B}$ the above reduces to the familiar frame formalism with local $\mathrm{SO}(d) \times \mathrm{SO}(d)$ invariance but for our present application an alternative gauge fixing is more convenient: using matrix notation, we set

$$
\kappa=\left(\begin{array}{cc}
2 G & 0 \\
0 & 2 G
\end{array}\right),
$$

and

$$
E=\frac{1}{2}\left(\begin{array}{cc}
1+B G^{-1} & 1-B G^{-1} \\
G^{-1} & -G^{-1}
\end{array}\right)
$$

Defining the Maurer-Cartan forms

$$
\left(E^{-1} \partial_{\mu} E\right)_{A}{ }^{B} \equiv\left(\begin{array}{cc}
Q_{\mu a}{ }^{b} & P_{\mu a}^{\bar{b}} \\
\bar{P}_{\mu \bar{a}}{ }^{b} & \bar{Q}_{\mu \bar{a}} \bar{b}
\end{array}\right)
$$

one finds that the $P_{\mu}$ transform as $\mathrm{GL}(d) \times \mathrm{GL}(d)$ tensors while the $Q_{\mu}$ transform as $\mathrm{GL}(d) \times \mathrm{GL}(d)$ connections:

$$
\delta_{\Lambda} Q_{\mu a}{ }^{b}=-D_{\mu} \Lambda_{a}^{b}, \quad \delta_{\Lambda} \bar{Q}_{\mu \bar{a}}{ }^{\bar{b}}=-D_{\mu} \bar{\Lambda}_{\bar{a}}{ }^{\bar{b}},
$$

where $D_{\mu} \Lambda_{a}{ }^{b}=\partial_{\mu} \Lambda_{a}{ }^{b}+\left[Q_{\mu}, \Lambda\right]_{a}{ }^{b}$, and analogously for the barred version. For the gauge choice (28) the explicit form of these connections reads, in matrix notation, 


$$
\begin{aligned}
Q_{\mu} & =-\frac{1}{2} \partial_{\mu}(G-B) G^{-1}, \\
\bar{Q}_{\mu} & =-\frac{1}{2} \partial_{\mu}(G+B) G^{-1} .
\end{aligned}
$$

We can now consider the Chern-Simons 3-form built from these connections:

$$
\mathrm{CS}_{\mu \nu \rho}(Q) \equiv \operatorname{Tr}\left(Q_{[\mu} \partial_{\nu} Q_{\rho]}+\frac{2}{3} Q_{[\mu} Q_{\nu} Q_{\rho]}\right),
$$

which transforms under Eq. (30) as

$$
\delta_{\Lambda} \mathrm{CS}_{\mu \nu \rho}(Q)=\partial_{[\mu} \operatorname{Tr}\left(\partial_{\nu} \Lambda Q_{\rho]}\right) .
$$

The barred formulas are analogous. Working out the ChernSimons-form for Eq. (31) one finds precisely Eq. (14), up to a global factor 3 . We thus define the 3-form curvature with Chern-Simons modification as

$$
\tilde{H}_{\mu \nu \rho} \equiv H_{\mu \nu \rho}-\frac{3}{2} \alpha^{\prime}\left(\mathrm{CS}_{\mu \nu \rho}(Q)-\mathrm{CS}_{\mu \nu \rho}(\bar{Q})\right) .
$$

In this formulation the $\mathrm{O}(d, d, \mathbb{R})$ invariance is manifestly realized without deformation. Rather, the $\mathrm{GL}(d) \times \mathrm{GL}(d)$ gauge symmetry is deformed, with the 2-form transforming according to the Green-Schwarz mechanism,

$$
\delta B_{\mu \nu}=\frac{1}{2} \alpha^{\prime} \operatorname{Tr}\left(\partial_{[\mu} \Lambda Q_{\nu]}\right)-\frac{1}{2} \alpha^{\prime} \operatorname{Tr}\left(\partial_{[\mu} \bar{\Lambda} \bar{Q}_{\nu]}\right) .
$$

Upon partial gauge fixing to $\mathrm{SO}(d) \times \mathrm{SO}(d)$ and field redefinition this transformation relates to the reduction of $\alpha^{\prime}$-deformed double field theory [17]. If we fully gauge fix $\mathrm{GL}(d) \times \mathrm{GL}(d)$ [as done above in order to obtain explicit forms like Eq. (31)] the $\mathrm{O}(d, d, \mathbb{R})$ transformations get deformed through compensating gauge transformations so that $B_{\mu \nu}$ starts transforming under $\mathrm{O}(d, d, \mathbb{R})$.

We finally discuss the $\mathbb{Z}_{2}$ invariance (22) of bosonic string theory in the frame formulation. It acts on the frame field as

$$
E \rightarrow Z^{T} E \tilde{Z}, \quad \tilde{Z} \equiv\left(\begin{array}{ll}
0 & 1 \\
1 & 0
\end{array}\right)
$$

The matrix $\tilde{Z}$ has the effect of exchanging the two GL $(d)$ factors or, equivalently, to exchange the role of unbarred and barred indices. Indeed, a quick computation shows that under Eq. (36) the Maurer-Cartan forms (29) transform as $P_{\mu} \leftrightarrow \bar{P}_{\mu}$ and $Q_{\mu} \leftrightarrow \bar{Q}_{\mu}$, in agreement with the explicit form (31). Thus, thanks to the relative sign in Eq. (34) the total Chern-Simons form is $\mathbb{Z}_{2}$ odd, which together with $B_{\mu \nu} \rightarrow-B_{\mu \nu}$ implies $\mathbb{Z}_{2}$ invariance.

We have shown that Green-Schwarz type mechanisms may be necessary even in classical string theory, which includes $\alpha^{\prime}$ corrections, in order to realize its global duality symmetries. While the original Green-Schwarz mechanism of Ref. [18] does modify the classical theory, in that case the need for an $\alpha^{\prime}$ deformation of gauge transformations follows by requiring cancellation of quantum anomalies. The observation that already invariance of the purely classical theory requires Green-Schwarz type mechanisms suggests that these may play a much more general role, perhaps in order to revisit $U$ duality in the presence of higher order corrections.

We thank J. Maharana and D. Marqués for helpful discussions. The work of O. H. is supported by the ERC Consolidator Grant "Symmetries \& Cosmology."

[1] C. Eloy, O. Hohm, and H. Samtleben (to be published).

[2] A. Sen, $O(d) \otimes O(d)$ symmetry of the space of cosmological solutions in string theory, scale factor duality and two-dimensional black holes, Phys. Lett. B 271, 295 (1991).

[3] G. Veneziano, Scale factor duality for classical and quantum strings, Phys. Lett. B 265, 287 (1991).

[4] J. Maharana and J. H. Schwarz, Noncompact symmetries in string theory, Nucl. Phys. B390, 3 (1993).

[5] K. A. Meissner, Symmetries of higher order string gravity actions, Phys. Lett. B 392, 298 (1997).

[6] N. Kaloper and K. A. Meissner, Duality beyond the first loop, Phys. Rev. D 56, 7940 (1997).,

[7] H. Godazgar and M. Godazgar, Duality completion of higher derivative corrections, J. High Energy Phys. 09 (2013) 140.

[8] W. Siegel, Superspace duality in low-energy superstrings, Phys. Rev. D 48, 2826 (1993).

[9] C. Hull and B. Zwiebach, Double field theory, J. High Energy Phys. 09 (2009) 099.

[10] O. Hohm, C. Hull, and B. Zwiebach, Generalized metric formulation of double field theory, J. High Energy Phys. 08 (2010) 008.

[11] O. Hohm, W. Siegel, and B. Zwiebach, Doubled $\alpha^{\prime}$-geometry, J. High Energy Phys. 02 (2014) 065.

[12] O. Hohm and B. Zwiebach, Green-Schwarz mechanism and $\alpha^{\prime}$-deformed Courant brackets, J. High Energy Phys. 01 (2015) 012.

[13] O. Hohm and B. Zwiebach, Double field theory at order $\alpha^{\prime}$, J. High Energy Phys. 11 (2014) 075.

[14] D. Marques and C. A. Nunez, T-duality and $\alpha^{\prime}$-corrections, J. High Energy Phys. 10 (2015) 084.

[15] O. Hohm, Background Independence and Duality Invariance in String Theory, Phys. Rev. Lett. 118, 131601 (2017).

[16] O. Hohm, Background independent double field theory at order $\alpha^{\prime}$ : Metric vs. frame-like geometry, Phys. Rev. D 95, 066018 (2017).

[17] W. H. Baron, J. J. Fernandez-Melgarejo, D. Marques, and C. Nunez, The Odd story of $\alpha^{\prime}$-corrections, J. High Energy Phys. 04 (2017) 078.

[18] M. B. Green and J.H. Schwarz, Anomaly cancellation in supersymmetric $D=10$ gauge theory and superstring theory, Phys. Lett. B 149, 117 (1984). 
[19] R. R. Metsaev and A. A. Tseytlin, Order $\alpha^{\prime}$ (two loop) equivalence of the string equations of motion and the $\sigma$-model Weyl invariance conditions: Dependence on the dilaton and the antisymmetric tensor, Nucl. Phys. B293, 385 (1987).
[20] O. Hohm and B. Zwiebach, T-duality constraints on higher derivatives revisited, J. High Energy Phys. 04 (2016) 101.

[21] O. Hohm and B. Zwiebach, Duality invariant cosmology to all orders in $\alpha^{\prime}$, Phys. Rev. D 100, 126011 (2019). 